\title{
Data Dissemination in Wireless Broadcast Channels: Network Coding versus Cooperation
}

\author{
Ivana Stojanovic, Zeyu Wu, Masoud Sharif, and David Starobinski \\ ECE Department, Boston University \\ Email: \{ivanas,bobtail,sharif,staro\}@bu.edu
}

\begin{abstract}
Network coding and cooperative diversity have each extensively been explored in the literature as a means to substantially improve the performance of wireless networks. Yet, little work has been conducted to compare their performance under a common framework. Our goal in this paper is to fill in this gap. Specifically, we consider a single-hop wireless network consisting of a base station and $N$ receivers. We perform an asymptotic analysis, as $N \rightarrow \infty$, of the expected delay associated with the broadcasting of a file consisting of $K$ packets. We show that if $K$ is fixed, cooperation outperforms network coding, in the sense that the expected delay is proportional to $K$ (and thus within a constant factor of the optimal delay) in the former case while it grows logarithmically with $N$ in the latter case. On the other hand, if $K$ grows with $N$ at a rate at least as fast as $(\log N)^{r}$, for $r>1$, then we show that the average delay of network coding is also proportional to $K$ and lower than the average delay of cooperation if the packet error probability is smaller than 0.36 . Our analytical findings are validated through extensive numerical simulations.
\end{abstract}

\section{INTRODUCTION}

Significant breakthroughs in wireless technology over the past decade have enabled implementation of third generation cellular systems capable of offering services traditionally more applicable to wireline networks. In particular, the growing use of cellular systems has lead to demand for broadcasting services requiring simultaneous data transmission to multiple users. Notable examples of such services include podcasting and distributions of software updates [1].

It has been recently shown that network coding provides the maximum achievable throughput gains for multicast and broadcast wired networks [2], [7], [8]. In wireless networks, network coding has been proposed for disseminating information to all receivers [3] and has been shown to provide excellent delay performance compared to round-robin scheduling, in particular, for large file sizes. In this context, the delay is defined as the average number of packet transmissions to transmit a file consisting of $K$ packets to all $N$ receivers. Network coding relies on the transmission of algebraic combinations of $K$ packets implying that the individual packets can be decoded only after the correct reception of at least $K$ combinations [2].

In this paper, we propose a cooperative transmission scheme based on round-robin scheduling as an alternative method that substantially reduces the error probability of the wireless link in re-transmissions of a packet and therefore improves the transmission delay. We compare the delay benefit of this

The results of this paper have been presented in part in 2007 Conference on Information Science and Systems (CISS). cooperative-based scheduling scheme with network coding which has not been done before. The proposed cooperative transmission scheme is done by exploiting a relatively small number of receivers who have already successfully decoded the packet to cooperatively transmit the packet to the rest of network. This amounts to a distributed multi-antenna system with substantial diversity gain benefit and reduction in the error probability [12] [13], [14].

Towards this end, we analyze and compare the delay performance of network coding and cooperation in a single-hop wireless network (i.e., downlink) composed of one base station and $N$ nodes. Single-hop means that all nodes can communicate directly with each other. In the cooperative scheme this translates to packets being simultaneously transmitted by the base station and a subset of nodes serving as relays. We provide closed-form asymptotic expressions for the average delay of these two schemes, as $N \rightarrow \infty$. In particular, for network coding, we show that if the number of packets $K$ of the file is fixed, then the average delay scales like $\log _{1 / p_{e}} N$ where $p_{e}{ }^{1}$ is the error probability of the link between the transmitter and any receiver. As the number of packets increases, even polylogarithmically with $N$, it is shown that the delay achieved by network coding is proportional to $K$. On the other hand, for cooperation, the average delay is proportional to $K$ for any $K$, and thus achieves performance within a constant factor of the optimal delay. This result holds even if a relatively small number of receivers are allowed to cooperate, in fact as few as $(\log N)^{r}$ for $r>1$, to achieve near-optimal delay performance.

Our results imply that when the number of packets $K$ is much smaller than $N$, the cooperative scheme outperforms network coding. However, as $K$ grows logarithmically with $N$ or faster, the average delay of network coding is within a factor of $1+\frac{1}{\log 1 / p_{e}}$ of the optimal delay, lower than the average delay of cooperation for the practical case $p_{e}<0.36$. Note that, for the sake of tractability, our analysis ignores overhead associated with the implementation of the studied schemes. Thus, our results should be interpreted as lower bounds on practical delay performance.

The rest of the paper is organized as follows. In Section II, we introduce our network model and underlying statistical assumptions. In Section III, we provide delay bounds for the baseline, traditional round-robin scheduling. In Section IV and $\mathrm{V}$, we respectively analyze the asymptotic performance

\footnotetext{
${ }^{1}$ In our analysis $p_{e}$ is assumed to be fixed independent of $N$.
} 
of random linear coding and cooperation for large networks and different regimes of file sizes. In Section VI, we present numerical results comparing the performance of the two strategies. We conclude the paper in Section VII.

\section{SySTEM MODEL}

We consider a cellular downlink consisting of a single antenna base station and $N$ single antenna receivers. We are concerned with the case where a single file, consisting of $K$ packets, is demanded by all the receivers in the network. We are interested in finding the average file transfer delay, defined as the minimum number of channel uses that guarantees complete download of the whole file by all nodes in the network.

Files are segmented into packets to be transmitted in time slotted manner, with a single packet transmission requiring one time slot. The transmission takes place over a time varying broadcast channel. The assumed channel is Rayleigh blockfading that changes independently from one block to the other. One channel block corresponds to one time slot and, thus, one packet transmission.

In wireless channels, packets are usually dropped when the channel goes into deep fade resulting in an outage. In particular, the outage happens when instantaneous channel capacity falls below the amount of information carried in the packet [11]. Therefore, we can model the channel from the base station to the $n$-th receiver as a random on/off channel, with parameter $p_{e}$ representing the probability of 'off' or outage state. Since fading is independent across time and users, the error events are similarly independent across time and users.

We assume that the network is homogeneous implying that the channels between any user and the base station have identical and independent distribution. In a cooperative scheduling scenario, analyzed in more detail in a subsequent section, users that have correctly received a packet can potentially aid the base station in future transmissions of the same packet. We assume that channels between any user acting as a transmitter and all users acting as receivers are independent and identically distributed, with the same distribution as the distribution of channels between the base station and any user. In our setup, we assume that each receiver has perfect knowledge of its own channel state toward the base station and users acting as transmitters, but the channel state information (CSI) is unknown to transmitters. In this paper, we are interested in characterizing the average delay for the regime of large $N$ and for various regime of $K$, i.e., $K$ fixed or growing with $N$.

It is quite clear that the lower bounds on the minimum delay for sending the $K$ packets to all users is $K$. In the next section, we show that the delay of a simple, plaintext round-robin transmission can be significantly worse than that of the lower bound.

\section{Round-RobIN SCHEDULING}

In this section, we consider a simple round-robin transmission scheme in which the base station sends each packet sequentially until every user is able to decode all the $K$ packets. We assume that the base station stops scheduling a certain packet $k$ once every node received it. All outstanding packets are cyclicly scheduled by the base station at regularly spaced time slots until all nodes successfully received the whole file. To simplify the analysis, we ignore the overhead of the control channel.

Denote by $D_{n}^{k}$ the number of transmissions of packet $k$ needed for user $n$ to receive packet $k$. The random variable $D_{n}^{k}$ is geometrically distributed with probability of success $\left(1-p_{e}\right)$. A packet transmission is considered successful once it is received by all nodes in the network. Let $D^{k}$ represent the number of transmissions of the packet $k$ until its successful reception by all users. Therefore, the $k$-th packet delay is given by $D^{k}=\max _{n=1, \ldots, N} D_{n}^{k}$. Since different packet transmissions are non-overlapping in the scheduling mode, the file completion delay is calculated as $D_{R R}=\sum_{k=1}^{K} D^{k}$.

Lower and upper bounds on the optimal expected completion delay for this policy are presented in [18], Proposition 1, using properties of stochastic ordering. This result is reproduced here, for completeness.

Proposition 3.1: Consider the time slotted broadcasting of a single $K$-packet file to all $N$ users. Under a round-robin scheduling, the expected delay, denoted by $\mathbb{E}\left[D_{R R}\right]$, is given by,

$$
\mathbb{E}\left[D_{R R}\right]=K \log _{1 / p_{e}}(N)+O(1) .
$$

for large $N$ and any $K$.

This result shows, that for large $N$, the delay of a roundrobin scheme in a noisy channel can be substantially worse than in a noiseless channel. In the next section, we show that this large gap, i.e., $\log _{1 / p_{e}} N$, can be reduced using random linear coding, but only for $K$ growing fast enough with $N$.

\section{RANDOM LINEAR CODING}

In this section we analyze the scaling law of the expected delay achieved by a random linear coding (RLC) policy. In a slot $i$, where a slot corresponds to the duration of a packet transmission, the transmitted packet is actually computed as a linear combination of all packets: $P[i]=\sum_{k=1}^{K} a_{k}[i] P_{k}$, such that $a_{k}[i] \in \mathbb{F}_{q}$ for each $k \in\{1, \ldots, K\}$ and where $q$ represents the size of the finite coefficient field $\mathbb{F}_{q}$ [9]. Coefficients $a_{k}[i]$ are chosen uniformly at random over the field $\mathbb{F}_{q}$ for each $k$.

Each receiver stores all correctly received packets up until a time it collects $K$ linearly independent combinations. The expected number of successful receptions before a user receives $K$ linearly independent combinations is upper bounded by $K q /(q-1)$ [4], [5], [6]. Notice also that it is impossible for a receiver to successfully decode a $K$-packet file with less than $K$ successful receptions. In this paper we assume that the upper bound is tight and made close to $K$ by choosing field size $q$ sufficiently large. We also ignore the communication overhead of network coding. Then, by standard linear coding arguments, the mean completion time of the $K$ packet file is equal to $\mathbb{E}\left[\max _{n=1, \ldots N} T_{n}\right]$, where $T_{n}$ is the minimum number of time slots before $K$ transmissions can be successfully 
decoded by node $n$. The random variable $T_{n}$ is the sum of $K$ independent geometric random variables and thus, $T_{n}$ has a negative binomial distribution with parameters $1-p_{e}$ and $K$. Denote the file transmission delay of the random linear coding policy by $D_{R L C}$. Then, the average file transmission delay is given by [3]:

$$
\begin{aligned}
& \mathbb{E}\left[D_{R L C}\right]=K \\
& \quad+\sum_{t=K}^{\infty}\left[1-\prod_{i=1}^{N}\left(\sum_{\tau=K}^{t}\left(\begin{array}{c}
\tau-1 \\
K-1
\end{array}\right) p_{e}^{\tau-K}\left(1-p_{e}\right)^{K}\right)\right]
\end{aligned}
$$

To gain more insight into behavior of the expected delay for large networks, we provide an asymptotic analysis of the delay in the regime of large $N$ and for different cases of file length $K$ where $K$ is fixed, $K$ grows logarithmically with $N$, and finally $K$ grows faster than logarithmically with $N$.

Theorem 4.1: Consider the setting of Proposition 3.1. Under randomized linear coding strategy and for large $N$, the scaling law of the average delay in sending the file of size $K$ packets to all $N$ users is given by,

1) For fixed $K$,

$$
\begin{aligned}
& \mathbb{E}\left[D_{R L C}\right]= \\
& \quad \log _{1 / p_{e}} N+(K-1) \log _{1 / p_{e}} \log N+o(\log \log N) .
\end{aligned}
$$

2) For $K$ growing $\log$ arithmically with $N$, i.e., $K=\log N$,

$$
\mathbb{E}\left[D_{R L C}\right]=\beta_{1} K+o(\log N),
$$

where $3.146 /\left(\log 1 / p_{e}\right) \leq \beta_{1} \leq 1+3.146 /\left(\log 1 / p_{e}\right)$.

3) For $K$ growing faster than $(\log N)^{r}$, where $r>1$,

$$
\mathbb{E}\left[D_{R L C}\right]=\beta_{2} K+o\left((\log N)^{r}\right),
$$

where $1 /\left(\log 1 / p_{e}\right) \leq \beta_{2} \leq 1+1 /\left(\log 1 / p_{e}\right)$.

Proof: We first obtain lower and upper bounds on the expected delay using the properties of stochastic ordering as in [18]. Next, given the upper and lower bounds on the expected delay we derive the asymptotic result for the first moment of $D_{R L C}$ for different regions of $K$ and $N$.

As mentioned $D_{R L C}=\max _{n=1, \ldots N} T_{n}$ where $T_{n}$ is the sum of $K$ independent geometric variables $T_{n}^{k}$, i.e., $T_{n}=\sum_{k=1}^{K} T_{n}^{k}$. Here $T_{n}^{k}$ has a geometric distribution with parameter $1-p_{e}$. An equivalent continuous random variable $X_{n}^{k}$ with the same mean has the pdf $f(x)=$ $\sum_{i=1}^{\infty} p_{e}^{i-1}\left(1-p_{e}\right) \delta(x-i)$ where $\delta(x)$ is the Dirac's delta function. The complementary cumulative distribution function (CCDF) of this variable is $\bar{F}(x)=p_{e}^{\lceil x\rceil-1}$, for $x>0$.

Now we consider an exponential random variable $Y_{n}^{k}$ with parameter $\lambda=\log 1 / p_{e}$ that has the CCDF of $\bar{F}_{Y_{n}^{k}}(x)=p_{e}^{x}$. We further define $Z_{n}^{k}=Y_{n}^{k}+1$ with CCDF of ${ }^{n} \bar{F}_{n}^{k}(x)=$ $\min \left(1, p_{e}^{x-1}\right)$. The CCDFs of $X_{n}^{k}, Y_{n}^{k}, Z_{n}^{k}$ clearly satisfy $\bar{F}_{Y_{n}^{k}}(x) \leq \bar{F}_{X_{n}^{k}}(x) \leq \bar{F}_{Z_{n}^{k}}(x)$ implying by definition the desired stochastic ordering of $Y_{n}^{k} \leq_{s t} X_{n}^{k} \leq_{s t} Z_{n}^{k}$. Note that the notation $X \leq_{s t} Z$ denotes that the random variable $X$ is stochastically dominated by the random variable $Z$.

Finally, we consider $X_{n}=\sum_{k=1}^{K} X_{n}^{k}, Y_{n}=\sum_{k=1}^{K} Y_{n}^{k}$ and $Z_{n}=\sum_{k=1}^{K} Z_{n}^{k}$. These variables have the same stochastic ordering $Y_{n} \leq_{s t} X_{n} \leq_{s t} Z_{n}$ leading to

$$
\mathbb{E}\left[\max _{n=1, \ldots N} Y_{n}\right] \leq \mathbb{E}\left[D_{R L C}\right] \leq \mathbb{E}\left[\max _{n=1, \ldots N} Z_{n}\right]
$$

where we used the fact that $\mathbb{E}\left[D_{R L C}\right]=\mathbb{E}\left[\max _{n=1, \ldots N} X_{n}\right]$.

Now, we evaluate the asymptotic behavior of the bounds. In fact, the distribution of $Y_{n}$ can be written as

$$
\bar{F}_{Y_{n}}(x)=\sum_{i=0}^{K-1} \frac{(\lambda x)^{i}}{i !} \exp ^{-\lambda x} .
$$

as $Y_{n}$ is the sum of $K$ exponentially distributed random variables. Therefore,

$$
\mathbb{E}\left[\max _{n=1, \ldots N} Y_{n}\right]=\frac{1}{\lambda} \int_{0}^{\infty}\left(1-\left(1-S_{K}(x) e^{-x}\right)^{N}\right) d x
$$

where $S_{K}(x)=\sum_{i=0}^{K-1} \frac{x^{i}}{i !}$ and $\lambda=\log 1 / p_{e}$. The asymptotic behavior of the integral in (4) is studied in [16] in a different context for large $N$. When $K$ is fixed, it is shown that the right hand side of (4) scales like,

$$
\frac{1}{\lambda}(\log N+(K-1) \log \log N+o(\log \log N)),
$$

which leads to a lower bound on the expected delay. We can also find an upper bound by evaluating $\mathbb{E}\left[\max _{n=1, \ldots N} Z_{n}\right]$ and noting that $\bar{F}_{Z_{n}}(x)=\bar{F}_{Y_{n}}(x-K)$ and $\mathbb{E}\left[\max _{n=1, \ldots N} Z_{n}\right]=$ $K+\mathbb{E}\left[\max _{n=1, \ldots N} Y_{n}\right]$. Therefore, we get

$$
\begin{aligned}
& \mathbb{E}\left[\max _{n=1, \ldots N} Z_{n}\right]=K \\
& \quad+\frac{1}{\lambda}(\log N+(K-1) \log \log N+o(\log \log N)) .
\end{aligned}
$$

Substituting (5) and (7) into (3), we obtain the first part of the theorem. The proofs of the second and third parts follow the same line as the proof of the first part, appealing arguments similar to those developed in [16] and [17] Section 5.7.5. We omit the proof for the sake of brevity.

Theorem 4.1 implies that when $K$ is fixed, the delay performance of RLC in a noisy channel can be much worse than that of the noiseless channels, even though the performance is $K$ times better than that of the round robin scheduling obtained in Section 3. As $K$ grows slowly with $N$, i.e., only logarithmically with $N$, the delay scales linearly with $K$ and the performance gap is at most $1+3.146 /\left(\log 1 / p_{e}\right)$. Finally, if $K$ grows faster than logarithmically with $N$, the expected delay of RLC is at most $1+1 /\left(\log 1 / p_{e}\right)$ worse than in a noiseless channel. Note that for $p_{e} \leq 0.36$, the expected delay of RLC is within a factor of 2 of the delay in a noiseless channel.

In the next section, we show that for fixed or slowly growing $K$ the performance gap between noisy and noiseless channels can be further reduced using cooperative transmissions.

\section{Cooperative Scheduling}

In this section we describe and analyze a cooperative scheduling strategy. The idea behind this strategy lies on the simple observation that a successful file download by all users in the scheduling mode requires repeated transmissions of the same packet. Therefore at the beginning of the second 
transmission of a packet, there exist additional spatially dispersed nodes that can decode the packet successfully with high probability. Therefore, these nodes can collaborate in transmitting the packet to the rest of the users. In particular, it is well known that by using space time coding, significant improvement on the error probability can be obtained using collaborative nodes [19]. The gain is due to the fact that the fading channels corresponding to the different transmitters, i.e., the base station and $M$ cooperative nodes, are independent leading to better error probability via space diversity.

The packet loss probability in point-to-point MIMO channels is commonly characterized through a diversity gain $d$ and a coding gain $\alpha$ as $p_{e}=\alpha \rho^{-d}$, where $\rho$ is the signal to noise ratio [19]. The diversity gain of multi-antenna transmitters have been well studied in the literature. In particular, for the case where $M+1$ transmitters and one antenna receiver, it is straightforward to show that the packet error probability $p_{e}=\alpha \rho^{-1}$ can be reduced to $p_{e}(M)=\beta \rho^{-(M+1)}$ with proper space time coding where $\alpha$ and $\beta$ are constants independent of the $\mathrm{SNR}^{2}$. This shows the significant reduction in the error probability in the second transmission can be achieved by exploiting the receivers that have decoded the packet in the first transmission of the packet.

Definition 5.1: Cooperative Scheduling (CS) is a strategy in which $K$ packets of a file $F$ are transmitted in two stages. For every packet, in the first stage, the base station transmits the packet until at least $M$ nodes can decode the packet. In the second stage, the packet is re-transmitted cooperatively by the base station and the $M$ nodes who have successfully decoded the packet.

It turns out that by letting $M$ grow slightly faster than $\operatorname{logarithmically~with~} N$, i.e., $(\log N)^{r}$ for any $r>1$, we can achieve most of the gain offered by cooperation and can reduce the average delay to within a factor two of that in a noiseless channel for any $K$.

The next theorem provides the scaling law of the expected delay using a cooperative scheduling.

Theorem 5.1: Consider the setting of Proposition 3.1. The average delay $\mathbb{E}\left[D_{C S}\right]$ in sending $K$ packets of a file to all $N$ users achieved by cooperative round-robin scheduling is given by:

$$
\mathbb{E}\left[D_{C S}\right]=2 K(1+o(1)),
$$

for large $N$ and any $K$ where $M$ grows as $(\log N)^{r}$ for any $r>1$ independent of $N$.

Proof: We show the result by proving that the total expected number of transmissions in each of the two stages is equal to $K(1+o(1))$. In the first stage, we obtain the expected number of transmissions $\mathbb{E}\left[D_{\text {stage } 1}^{k}\right]$ in order to have at least $M=(\log N)^{r}$ users successfully decode the $k^{\prime}$ th packet for $r>1$. Then, we define $\mathbb{E}\left[D_{\text {stage }}\right]$ as a total time spent in stage 1 such that each of $K$ packets is successfully transmitted to at least $M$ nodes. Letting $N_{s}^{k}$ be the number of users that have successfully received packet $k$ after its first transmission, we get,

\footnotetext{
${ }^{2}$ Here $\alpha$ and $\beta$ only depend on the space time code and the geometry of the channel, i.e., the distances between the transmitters and the receiver.
}

$$
\begin{aligned}
\mathbb{E}\left[D_{\text {stage } 1}^{k}\right] & \leq \mathbb{P}\left\{N_{s}^{k} \geq M\right\}+\log _{1 / p_{e}} N \times \mathbb{P}\left\{N_{s}^{k}<M\right\} \\
& \leq 1+\log _{1 / p_{e}} N \times \mathbb{P}\left\{N_{s}^{k}<M\right\}
\end{aligned}
$$

where we used the fact that $\mathbb{E}\left[D_{\text {stage } 1}\right]$ is bounded by $\log _{1 / p_{e}} N$ using the result of Proposition 3.1. We can further prove that for $M=(\log N)^{r}$,

$$
\begin{aligned}
\mathbb{P}\left\{N_{s}^{k}<M\right\} & =\sum_{i=0}^{M-1}\left(\begin{array}{c}
N \\
i
\end{array}\right) p_{e}^{N-i}\left(1-p_{e}\right)^{i} \\
& =e^{-N \log 1 / p_{e}+o(N)},
\end{aligned}
$$

leading to $\mathbb{E}\left[D_{\text {stage } 1}^{k}\right]=1+o(1)$. Equation (7) can be obtained by first upper bounding $\left(1-p_{e}\right)^{i}$ with 1 and then replacing the leftover summation by $M$ times its maximal element achieved at $i=M=1$. Hence, the total average time spent in stage 1 is $\mathbb{E}\left[D_{\text {stage } 1}\right]=K(1+o(1))$.

In the second stage the cooperation of the base station and $M$ nodes reduces the probability of error from $p_{e}=\alpha \rho^{-1}$ to $p_{e}(M)=\beta \rho^{-(M+1)}=\left(\gamma p_{e}\right)^{M+1}$, where $\gamma=\frac{\beta^{1 /(M+1)}}{\alpha}$ takes into account the space-time coding gains $\alpha$ and $\beta$. Note that for fixed and finite $\alpha$ and $\beta, M=(\log N)^{r}$ and $N \rightarrow \infty$ we have $\gamma \rightarrow \frac{1}{\alpha}$. The minimum second stage delay is $K$, which is easily justified by noting that the probability of having to transmit each packet to at least one user in the second stage becomes one for fixed $p_{e}$ and $N \rightarrow \infty$. Furthermore, a delay of a transmission of $k$ 'th packet to all users in the second stage, $D^{k}(M)$, is a maximum of $N$ geometrically distributed random variables leading to the following bound on the expected second stage delay, $\mathbb{E}\left[D_{\text {stage } 2}\right]$ :

$$
\begin{aligned}
\mathbb{E}\left[D_{\text {stage } 2}\right] & =\mathbb{E}\left[\sum_{k=1}^{K} D^{k}(M)\right] \\
& =K \sum_{i=1}^{\infty} \mathbb{P}\left\{D^{k}(M) \geq i\right\} \\
& =K \sum_{i=1}^{\infty}\left(1-\left(1-\left(\gamma p_{e}\right)^{(M+1)(i-1)}\right)^{N}\right) \\
& =\frac{K}{M} \sum_{i=1}^{\infty} M \mathbb{P}\left\{D_{\gamma}^{k} \geq(M+1)(i-1)+1\right\} \\
& \leq K \frac{M-1}{M}+\frac{K}{M} \sum_{i=1}^{\infty} \mathbb{P}\left\{D_{\gamma}^{k} \geq i\right\} \\
& =K \frac{M-1}{M}+\frac{1}{M} \mathbb{E}\left[D_{R R}\right] \\
& =K+O\left(\frac{K}{M} \log _{\frac{1}{\gamma p_{e}}} N\right) .
\end{aligned}
$$

Equation (8) is obtained by calculating the CCDF of the maximum of $N$ geometrically distributed random variables with the probability of success $1-p_{e}(M)=1-\left(\gamma p_{e}\right)^{M+1}$. Equation (9) rewrites the same expression in terms of the summation of CCDFs of the maximum of $N$ geometrically distributed variables but with the probability of success $(1-$ $\gamma p_{e}$ ). The new variable $D_{\gamma}^{k}$ is equivalent to the delay of the $k$ th packet in the round-robin policy when communicating over 
a channel with probability of being in "on" state $\left(1-\gamma p_{e}\right)$. Inequality (10) is obtained by adding terms $\mathbb{P}\left\{D_{\gamma}^{k} \geq j\right\}, j \in$ $\{(M+1)(i-1)+2, \ldots,(M+1) i\}$ for each $i \in\{1,2, \ldots, \infty\}$ that are absent in the summation of Eq. (9). Equation (11) is obtained by the definition of the expected delay of the roundrobin policy and finally, the last equation is obtained by using the result of Proposition 3.1.

This results show that the expected delay for the second stage is also equal to $K(1+o(1))$ for the case where $M$ grows like $(\log N)^{r}$ for $r>1$.

Therefore,

$$
\mathbb{E}\left[D_{C S}\right]=\mathbb{E}\left[D_{\text {stage } 1}\right]+\mathbb{E}\left[D_{\text {stage } 2}\right]=2 K(1+o(1)),
$$

which completes the proof.

This theorem shows that the expected delay in a noisy channel achieved by cooperative transmission is asymptotically worse than that of noiseless channels by a factor of two in the regime of large $N$ and for any $K$. It is worth mentioning that this result is achieved using a relatively small number of cooperative nodes proportional to $(\log N)^{r}$ for $r>1$. Using more cooperative nodes, we can not further improve the expected delay beyond $2 K$. However, it does improve the convergence rate of the expected delay to $2 K$.

As mentioned, the diversity gain promised in the second round of transmission can be obtained via space time codes. In this scheme, the transmitters do not have the knowledge of the channel state of the users, however, every node expecting the packet in the second step needs to estimate its own channel to the base station and the $M$ other cooperative transmitters. It is also worth mentioning that since $M$ is relatively small and the channel estimation can be done in parallel for all receiving users, the corresponding overhead of each packet may be made negligible. Furthermore, since the probability of having a receiver act as a relay is $O(\log (N) / N)$, the average transmit power of each node (except for the base station) tends to zero. Finally, since the cooperative nodes are spatially dispersed, some sort of control messages has to be exchanged among all cooperating nodes in order to ensure proper spacetime encoding. In our analysis we did not take into account the delay cost associated with these overheads.

\section{COMPARISON AND NUMERICAL RESULTS}

In this section we present simulation results comparing the performance of the various schemes discussed in the paper, for various settings of the parameters $N, K, M$, and $p_{e}$.

In Figure 1 and Figure 2 we compare the average transmission delay of different schemes in the same setting as analyzed in [3]. Figure 1 shows that both network coding and cooperative scheduling offer significant average delay gains as compared to the baseline round-robin scheduling. The figure also shows that cooperation can achieve significant reduction in the expected delay by using as few as two cooperating nodes. Increasing the number of cooperating nodes to $M=(\log N)^{2}$, brings in the average cooperation delay below the delay of random linear coding and close to the delay of optimal cooperative scheduling as $N$ increases. Furthermore, cooperative scheduling with an unlimited number of cooperating nodes almost consistently outperforms random linear coding as it quickly approaches the minimum of $2 K$.

Figure 2 depicts the ratio of the average delay of cooperation to that of coding for highly lossy links with packet error probability $p_{e}=1 / 2$. For $M=\alpha \log N$, where $\alpha=\left(\log \frac{1}{p_{e}}\right)^{-1}$, cooperation outperforms RLC for files roughly smaller then 30 packets and a network size in the range of $100-500$ receivers. In the regime of small file transmissions, broadcast network applications of interest are, for example, emergency message distributions or small software updates. Increasing $M$ to $(\log N)^{2}$, we see that cooperation approaches its best possible delay of $2 K$ and consistently outperforms RLC over the packet range of interest. The ratio is growing rather slowly with the number of packets, so there must be a rather large file size to make random linear code eventually do better. By our analysis this ratio can approach at most two.

Figure 3 has the same setting as Figure 2, except that performance is compared for higher quality links with $p_{e}=0.1$. This situation fits better in practical wireless communication environments. Even though cooperation is within its optimal mean delay of $2 K$, RLC generally better utilizes the higher quality channel and performs better for almost all file lengths, except for very short files of few packets. As noted before the maximum ratio between the performance of the two schemes is at most two.

Figure 4, obtained for the case $K=10$ and $N=100$, shows that random coding performs better at low loss probability while cooperation is better at high loss probability, when using enough relays. We also note that when the number is relays is large enough, then the average delay of cooperative scheduling remains remains fixed at a value of $2 K$ over a wide range of packet loss probabilities. Both cooperative scheduling and random linear coding significantly outperform round-robin, sometimes by a factor as large as four.

\section{CONCLUSION}

In this paper we analyzed and compared delay performance of network coding and cooperative diversity under the common framework. Specifically, we analyzed the expected file completion delay of a $K$-packet file broadcast to $N$ users in a single-hop wireless network as $N \rightarrow \infty$. The results show that no technique is superior to the other in all regimes of different file lengths $K$.

In the regime of large file transmissions where $K$ grows at least as fast as $(\log N)^{r}, r>1$, random linear coding achieves better performance at a small packet loss probability. Its delay is within a factor of two of the optimal delay $K$, whereas the average cooperation delay scales asymptotically as $2 K$. In this regime, the average delay of random linear coding grows with the packet loss probability, whereas the average delay of cooperative scheduling delay remains constant provided that a sufficient number of nodes is allowed to cooperate. On the other hand, in the fixed $K$ regime, cooperation outperforms network coding. In this regime, network coding delay grows logarithmically with network size $N$, while cooperation retains its near-optimal delay of $2 K$.

In summary, the results indicate that in order to achieve near-optimal expected delay in both file length regimes and a 
small packet loss probability, one should choose network coding when broadcasting large files and cooperative scheduling for transmission of smaller files.

\section{REFERENCES}

[1] W. Xiao and D. Starobinski, " Poster Abstract: Exploiting MultiChannel Diversity to Speed Up Over-the-Air Programming of Wireless Sensor Networks", Proc. ACM SenSys 2005, San Diego, California, pp. 292-293, Nov. 2005.

[2] S. Y. R. Li, W. Yeung and N. Cai, "Linear Network Coding " IEEE Trans. Information Theory, vol.49, pp.371-381, Feb. 2003.

[3] A. Eryilmaz, A. Ozdaglar, and M. Medard , " On Delay Performance Gains from Network Coding," Proc. of Conference on Information Sciences and Systems (CISS), 2006.

[4] T. Ho, D. R. Karger, M. Medard, R. Koetter, " Network coding from a network flow perspective," Proc. of IEEE International Symposium on Information Theory (ISIT), 2003.

[5] S. Jaggi, P. A. Chou, K. Jain, " Low complexity algebraic multicast network codes," Proc. of IEEE International Symposium on Information Theory (ISIT), 2003.

[6] T. Ho, R. Koetter, M. Medard, D. R. Karger and M. Effros, "The Benefits of Coding over Routing in a Randomized Setting," Proc. of IEEE International Symposium on Information Theory (ISIT), 2003.

[7] S. Katti, D. Katabi, W. Hu, H. S. Rahul, and M. Medard, "The importance of being opportunistic: practical network coding for wireless environment", Proc. of Allerton Conference on Communication, Control and Computing, 2005.

[8] Y. Wu, "Network coding for multicasting," PhD. Thesis, Princton, 2005.

[9] T. Ho, "Networking from a network coding perspective," PhD. Thesis, MIT, 2004.

[10] B. Arnold, N. Balakrishnan and H. N. Nagaraja, A first course in order statistics, John Wiley and Sons, 1992.

[11] L. Zheng and D. N. C. Tse, "Diversity and Multiplexing: A Fundamental Tradeoff in Multiple Antenna Channels," IEEE Trans.Info.Theory, vol.IT-49, pp.1073-1096, May 2003.

[12] V. Tarokh, H. Jafarkhani, and A. R. Calderbank, " Space-Time Block Coding for Wireless Communications: Performance Results," IEEE Journal on Selected Areas in Communications, Vol. 17: No. 3, pp. 451-460, Mar. 1999.

[13] J. N. Laneman and G. W. Wornell " Distributed Space-Time Coded Protocols for Exploiting Cooperative Diversity in Wireless Networks," IEEE Trans. Inform. Theory, vol. 49, no. 10, pp. 2415-2525, Oct. 2003.

[14] J. N. Laneman, D. N. C. Tse, and G. W. Wornell, " Cooperative Diversity in Wireless Networks: Efficient Protocols and Outage Behavior," IEEE Trans. Inform. Theory, vol. 50, no. 12, pp. 3062-3080, Dec. 2004.

[15] S. Ross, Stochastic Processes, Wiley, 1996.

[16] M. Sharif and B. Hassibi, " Delay considerations for opportunistic scheduling in broadcast fading channels," IEEE Trans. Wireless Comm, vol. 6, issue 9, pp. 3353 - 3363, Sept. 2007.

[17] M. Sharif, "Broadband Wireless Broadcast Channels: Throughput, Performance and PAPR Reduction," PhD. Thesis, California Institute of Technology, 2005.

[18] D. Starobinski, W. Xiao, X. Qin, A. Trachtenberg, " Near-Optimal Data Dissemination Policies for Multi-Channel, Single Radio Wireless Sensor Networks," IEEE INFOCOM 2007, to appear.

[19] D. Tse and P. Viswanath, Fundamentals of Wireless Communication, Cambridge University Press, May 2005.

[20] I. Stojanovic, M. Sharif, D. Starobinski, "Data Dissemination in Wireless Broadcast Channels: Network Coding or Cooperation," Proc. of Conference on Information Sciences and Systems (CISS), 2007.

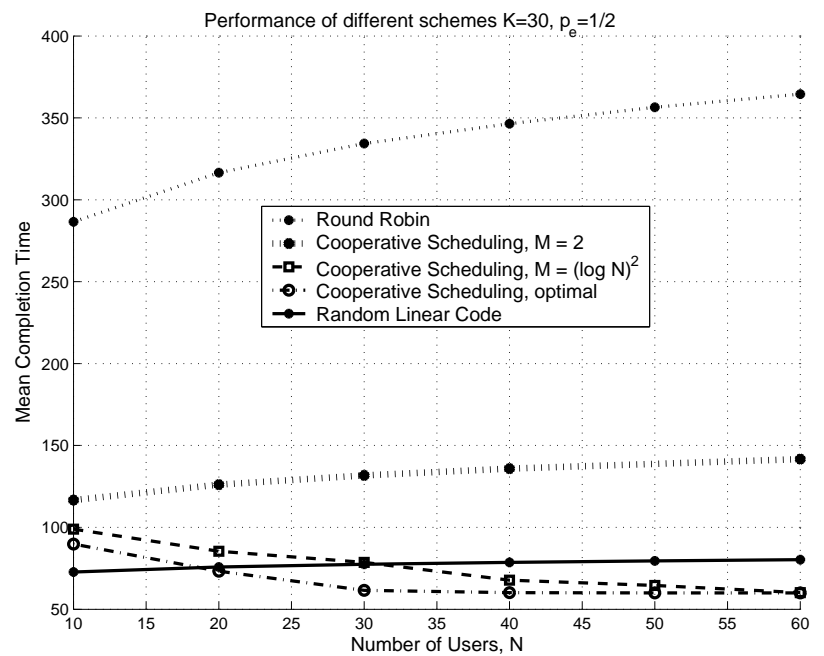

Fig. 1. Expected delay of different transmission strategies for $p_{e}=$ $1 / 2, K=30$.

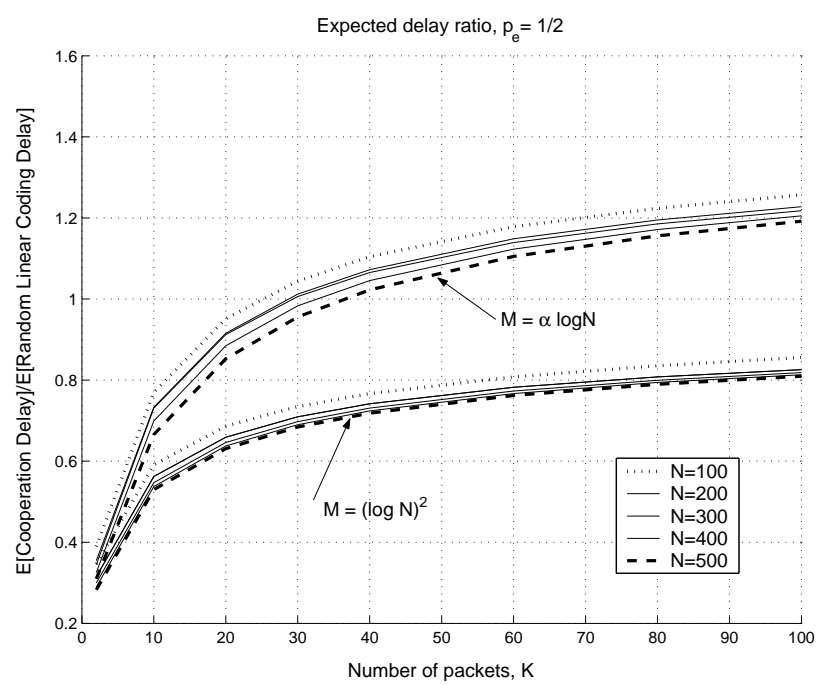

Fig. 2. Ratio of the expected file completion delay of the cooperative scheduling to the random linear coding at $p_{e}=1 / 2$ for different sizes of cooperation set $M$, and $\alpha=\left(\log \frac{1}{p_{e}}\right)^{-1}$. Note that all the ratio curves follow the regular trend, i.e. at fixed $K$ the ratio decreases as the number of users grows from $N=100$ to $N=500$. 


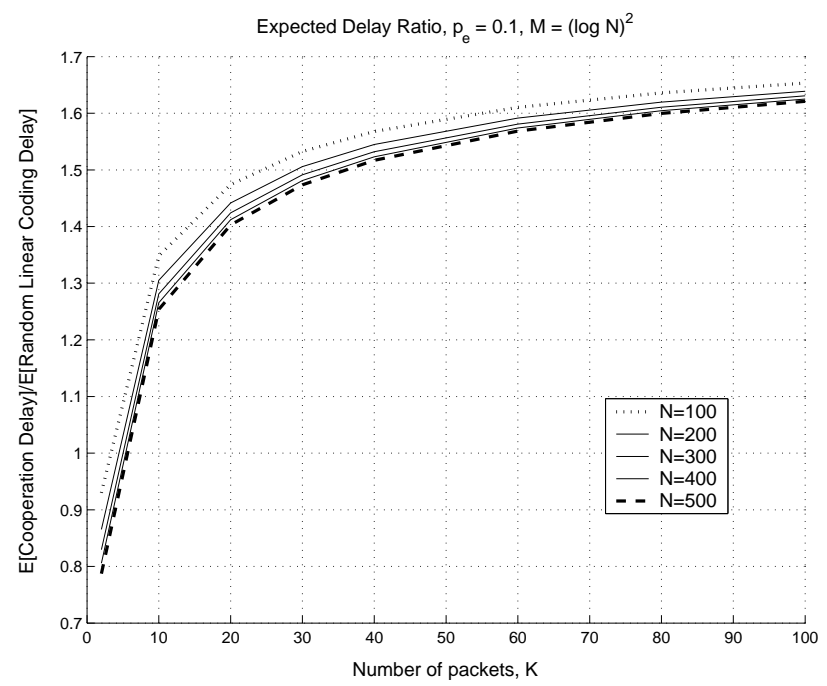

Fig. 3. Ratio of the expected file completion delay of the cooperative scheduling to the random linear coding policy at $p_{e}=0.1$ and $M=$ $(\log N)^{2}$. Note that all the ratio curves follow the regular trend, i.e. at fixed $K$ the ratio decreases as the number of users grows from $N=$ 100 to $N=500$.

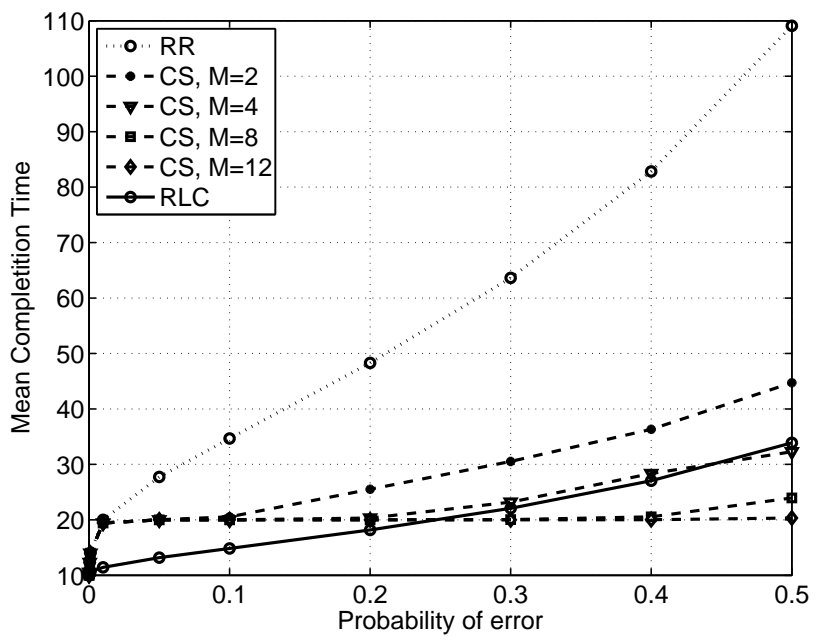

Fig. 4. Average file completion delay as a function of the probability of error at $K=10$ and $N=100$ for the three schemes: $R R$ (roundrobin), $C S$ (cooperative scheduling) and ( $R L C)$ random linear coding. 